УДК 656.025: 519.17

08.00.00 Экономические науки

МАТЕМАТИЧЕСКАЯ МОДЕЛЬ ЗАДАЧИ
ОРГАНИЗАЦИИ МАРШРУТОВ В
КРУПНОМАСШТАБНЫХ ТРАНСПОРТНЫХ
СЕТЯХ С ПРИМЕНЕНИЕМ МЕТОДОВ
МНОГОКРИТЕРИАЛЬНОЙ
ОПТИМИЗАЦИИ $^{1}$

Павлов Дмитрий Алексеевич

к.ф.-м.н., доцент

РИНЦ SPIN-код, 8822-5089

dp.logic@gmail.com

\author{
Яхонтова Ирина Михайловна \\ к.э.н., доцент \\ Кубанский государственный аграрный \\ университет, г. Краснодар, Россия
}

Большинство задач планирования и организации транспортных маршрутов сводится к решению оптимизационных задач на графах в многокритериальных постановках, для которых единственное оптимальное решение отсутствует. В условиях многокритериальности возникает необходимость вместо оптимума искать множество альтернатив. Качество допустимых решений оценивается векторной целевой функцией. В работе предлагается исследовать задачу с использованием особого класса графов предфрактальных, позволяющих естественным образом описать структуру иерархии территориальных связей, а так же дают возможность учитывать структурную динамику в плане роста системы. Строится многокритериальная математическая постановка задачи о покрытии предфрактального графа простыми пересекающимися цепями, к которой сводится исследуемая задача организации маршрутов в крупномасштабных транспортных сетях. Сформулированы и включены в модель в виде критериев основные социальные и экономические требования, предъявляемые к транспортной системе

Ключевые слова: ПРЕДФРАКТАЛЬНЫЙ ГРАФ, КРУПНОМАСШТАБНАЯ ТРАНСПОРТНАЯ СЕТЬ, ДИСКРЕТНАЯ МНОГОКРИТЕРИАЛЬНАЯ ОПТИМИЗАЦИЯ, СТРУКТУРНАЯ ДИНАМИКА

Doi: 10.21515/1990-4665-133-092
UDC 656.025: 519.17

Economics

\section{MATHEMATICAL MODEL OF THE PROBLEM OF ROUTE ORGANIZATION IN LARGE-SCALE TRANSPORT NETWORKS WITH THE APPLICATION OF MULTI- CRITERIAL OPTIMIZATION METHODS}

Pavlov Dmitriy Alexeevich

Cand.Phys.-Math.Sci., associate professor

SPIN-code, 8822-5089

dp.logic@gmail.com

Yahontova Irina Mihaylovna

Cand.Econom.Sci., associate professor

Kuban state agrarian University, Krasnodar, Russia

Most of the tasks of planning and organizing transport routes are pointed to solving optimization problems on graphs in multi-criteria statements, for which the only optimal solution is missing. In conditions of multicriteria, it becomes necessary to search for a set of alternatives instead of an optimum. The quality of the admissible solutions is estimated by the vector objective function. The article proposes to investigate the problem using a special class of graphs -

prefractal graphs, which allow describing in a natural way the structure of the hierarchy of territorial links, as well as enable to take into account structural dynamics in terms of system growth. A multicriteria mathematical formulation of the problem of covering a prefractal graph by simple intersecting chains is constructed, to which the investigated problem of organizing routes in large-scale transport networks reduces. The main social and economic requirements for the transport system are formulated and included in the model in the form of criteria

Keywords: PREFRACTAL GRAPH, LARGESCALE TRANSPORT NETWORK, DISCRETE MULTI-CRITERIAL OPTIMIZATION, STRUCTURAL DYNAMICS

\footnotetext{
${ }^{1}$ Исследование выполнено при финансовой поддержке РФФИ в рамках проекта 17-06-00282 a.
} 
Развитие экономических связей внутри любой страны и между странами предъявляет новые требования к планированию и организации маршрутов перевозок грузов и пассажиров в крупномасштабных транспортных сетях. Основной целью планирования является минимизация транспортных издержек. Однако при планировании оптимальных перевозок следует подходить не только с учетом экономических, но и общественных требований [1] которые, как правило, противоречат друг другу.

На основе математической модели возможна постановка оптимизационной задачи, решение которой позволяет точно оценить сложившуюся ситуацию и провести планирование дальнейших действий. Одним из самых эффективных способов проектирования и организации транспортных маршрутов являются дискретные оптимизационные модели на графах и сетях. Поиск решений оптимизационных задач на графах осуществляется специализированными алгоритмами. Качество поиска решения задачи алгоритмом оценивается его трудоемкостью или вычислительной сложностью [2]. Большинство точных алгоритмов решения оптимизационных задач на графах имеют не полиномиальную вычислительную сложность [2]. С ростом размерности задачи полиномиальные алгоритмы становится предпочтительнее экспоненциальных [2]. Таким образом, исследование задач с большим набором входных данных, в качестве которых выступает структура крупномасштабной транспортной сети, является актуальной проблемой и требует эффективных алгоритмов решений подобных задач.

В работе предлагается новый поход при планировании и организации транспортных маршрутов в крупномасштабных транспортных сетях, который основан на использовании в качестве формализованной модели транспортной сети [3], особого класса структур, называемых фрактальными графами [4]. Предфрактальные графы представляют собой 
симбиоз графа и фрактала, с присущими свойствами фракталов: самоподобием, дробной фрактальной размерностью, масштабной инвариантностью [5].

Интеграционные процессы и развитие экономических связей по истечению времени изменяет структуру транспортных сетей. Подобного рода изменения могут быть описаны простейшими теоретико-графовыми операциями: добавление (удаление) и стягивание ребра или вершины. Для описания постоянных изменений в структуре системы вводится понятие структурной динамики [6]. Одним из возможных правил, задающих структурную динамику, является процесс построения фрактальных графов.

Оговоримся заранее, что недостающие определения и термины можно найти в работах по теории графов [7] и предфрактальных графов [4].

Понятие предфрактального графа вводится индуктивно.

Обозначим через $H=(W, Q)$ - конечный связный $n$-вершинный граф с множеством вершин $W$ и множеством ребер $Q$, который назовем затравкой.

В основе порождения предфрактального графа лежит операция замещение вершины затравкой (3В3), как обобщенная процедура расщепления вершины графа [7].

Определим поэтапный процесс выполнения операции 3В3. На этапе $s=1$ графу $G_{1}=\left(V_{1}, E_{1}\right)$ соответствует затравка $H=(W, Q)$. На этапе $s=2$ все вершины графа $G_{1}$ замещаются затравкой. Далее, на каждом следующем этапе $s=1,2, \ldots, l$, к вершинам применяется операция 3В3, в результате по завершению этапа $l$ получаем граф $G_{l}=\left(V_{l}, E_{l}\right)$, который назовем предфрактальным.

Предфрактальный граф будем обозначать $G_{L}=\left(V_{L}, E_{L}\right)$, где $V_{L}-$ множество вершин, а $E_{L}-$ множество ребер. Ранг $L$ предфрактального 
графа $G_{L}=\left(V_{L}, E_{L}\right)$ определяет число этапов порождения графа. Процесс порождения предфрактального графа $G_{L}$ является процессом построения последовательности предфрактальных графов $G_{1}, G_{2}, \ldots, G_{L}$, называемой

$$
G=(V, E)
$$

траекторией. Фрактальный граф определяется бесконечной траекторией.

Предфрактальный граф $G_{L}=\left(V_{L}, E_{L}\right)$ является взвешенныл, если каждому его ребру $e^{(l)}=E_{L}$ поставлено в соответствие число $w\left(e^{(l)}\right) \in\left(\theta^{l-1} a, \theta^{l-1} b\right)$, где $l=1, L$ - ранг ребра, $a>0$ и $\theta<\frac{a}{b}$.

Обобщением процесса порождения предфрактального графа является случай, когда вместо одной затравки Н для замещения выбираются затравки из множества $\mathrm{H}=\left\{H_{\downarrow} \mathbf{1}, H_{\downarrow} 2, \ldots, H_{\downarrow} T\right\}$, где в качестве затравки может выбираться не только обыкновенный граф, но и мультиграф [7].

Рассмотрим карту сети дорог в определенном порядке, начиная с более мелкого масштаба (в масштабе континента или страны), заканчивая более крупным масштабом города или населенного пункта. В основе модели крупномасштабной транспортной сети лежит принцип иерархической организации территорий. При исследовании крупномасштабной транспортной сети в масштабах страны на первом этапе рассмотрим дороги связывающие округа. В качестве автомобильных дорог на этом этапе выступают магистрали и федеральные трассы. На втором этапе, в масштабе округов, рассмотрим сеть дорог соединяющих субъекты округов (области, республики, края). Далее, на третьем этапе при уменьшении масштаба последовательно рассматриваются дороги, связывающие определенные районы выбранного округа. Аналогично, при рассмотрении транспортной сети в масштабе района нас интересуют 
только дороги, соединяющие населенные пункты этого района. На последнем этапе рассматриваются дороги в масштабе населенных пунктов.

Процесс рассмотрения структуры транспортной сети в указанном порядке подобен траектории построения предфрактального графа, порожденного множеством затравок $\mathrm{H}=\left\{H_{\downarrow} 1, H_{\downarrow} 2, \ldots, H_{\downarrow} T\right\}, T \geq 2$.

Общая постановка задачи многокритериальной дискретной оптимизации [8] состоит из описаний условий, определяющих конечное или счетное множество допустимых решений $X=\{x\}$, и заданной на этом множестве векторно целевой функции (ВЦФ):

$$
F(x)=\left(F_{1}(x), F_{2}(x), \ldots, F_{N}(x)\right), F_{i}(x) \rightarrow e x t r, i=1, \ldots, N .
$$

Общей проблемой нахождения для дискретных многокритериальных задач является нахождение множества альтернатив (MA). Необходимость находить МА возникает в следствии того, что в самых различных областях человеческой деятельности принимаемое решение приходится оценивать многими критериями качества. Тогда в общем случае появляется ситуация, когда для каждого решения существуют альтернативы, т. е. другие решения, каждые из которых лучше рассматриваемого хотя бы по одному критерию.

Рассматриваются следующие виды МА:

1. Паретовское множество (ПМ). Элемент $\tilde{x} \in X$ называется недоминируемым или паретовским оптимумом (ПО), если не существует такого допустимого решения $x^{*} \in X$, что выполняются неравенства $F_{\gamma}\left(x^{*}\right) \leq F_{\gamma}(\widetilde{x}), \quad \gamma=1, \ldots, N$, среди которых хотя бы одно является строгим. Множество всех ПО называется ПМ и обозначается $\widetilde{X}$.

2. Полное множество альтернатив (ПМА) определяется как

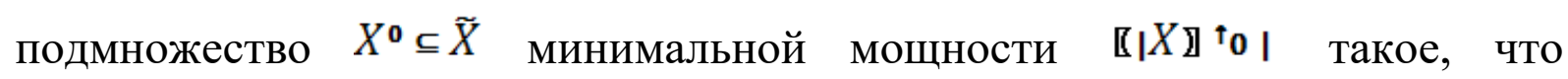
$F\left(X^{0}\right)=F(X)$, где $F\left(X^{*}\right)=\left\{F(x): x \in X^{*}\right\}_{\forall} X^{*} \in X$. 
Среди всех видов МА определенное ПМА является наиболее интересным математическим объектом, поскольку представляет собой обобщение классического понятия «решение оптимизационной задачи».

3. Лексикографическое множество альтернатив (ЛМА) состоит из лексикографических оптимумов (ЛО), определяемых следующим образом. Элемент $x^{0} \in \widetilde{X}$ называется ЛО, если существует такая перестановка $\gamma^{\mathbf{0}}=\tau_{1}, \ldots, \tau_{r}, \ldots, \tau_{N}$ чисел $1,2, \ldots, \mathrm{N}$, что для всякого $\tilde{x} \in \tilde{X}$ выполняется одно из двух условий:

a) $F_{\tau_{r}}(\widetilde{\chi})=F_{\tau_{r}}\left(x^{0}\right), \quad r=1, \ldots, N$;

б) существует $i \in\{1, \ldots, N\}$ такое, что $F_{\tau_{l}\left(x^{0}\right)<F_{\tau_{l}}(\widetilde{x})}$ и $F_{\tau_{l}(\widetilde{X})}=F_{\tau_{r}}\left(\chi^{0}\right), r=1, \ldots, l-\mathbf{1}$.

Пусть $\mathrm{B}=\{\beta\}-$ множество всех перестановок $N$ ! чисел $1, \ldots, N$. Для каждой перестановки $\beta \in \mathrm{B} \quad$ существует непустое множество $\tilde{x}_{\beta} \subseteq X$ ЛО, которые определены согласно соотношениям а) и б). Пусть $\tilde{x}_{\text {lex }}=\bigcup_{\beta \in \mathrm{B}} X_{\beta}$ множество всех ЛО данной $N$-критериальной информации. Тогда ее ЛМА определяется как подмножество $x^{0}$ lex $\subseteq \widetilde{x}_{l e x}$, имеющее наименьшую мощность при выполнении равенства $\mathbb{E}\left(x^{0} \rrbracket_{l e x}\right)=F\left(x \rrbracket_{l e \Xi}\right)$. Иначе говоря, для всякого ПМА $X^{0} \subseteq \widetilde{X}_{\varpi}$ соответствующее ему ЛМА $X^{0}{ }_{\text {lex }}$ определяется единственным образом в виде пересечения $X^{0} \cap \widetilde{X}_{l e x}=X^{0}$ lex.

При фиксированном наборе системы ограничений и параметров ВЦФ принято говорить о индивидуальной задаче.

Рассмотрим многокритериальную математическую постановку задачи о покрытии [9] предфрактального графа простыми пересекающимися цепями, к которой сводится задача организации маршрутов в крупномасштабных транспортных сетях. 
Пусть дан взвешенный предфрактальный граф $G_{L}=\left(V_{L}, E_{L}\right)$ порожденный затравкой $H=(W, Q),|W|=n,|Q|=q$.

Покрытием графа $G_{L}$ назовем подграф $x=\left(V_{L}, E_{x}\right)$, $E_{x}$ EMBED Equation. 3 国团 $E_{L}$, построенный из множества простых цепей $\left\{C_{1}, C_{2}, \ldots, C_{k}, \ldots, C_{K}\right\}$, где между двумя любыми вершинами из покрытия имеется простая цепь []. Множество всех покрытий $x$ обозначим через $X$. Покрытие $x=\left(V_{L}, E_{x}\right)$ - связный подграф графа $G_{L}$. Покрытие состоит из простых цепей пересекающихся по вершинам либо ребрам.

Максимальной назовем кратчайшую цепь не являющуюся подцепью никакой другой кратчайшей цепи [7].

В предфрактальном графе $G_{L}$ простую цепь будем называть $i$ смешанной цеепью $C^{i}$, если она содержит ребра $i$ различных рангов.

На множестве покрытий $x \in X$ графа $G_{L}=\left(V_{L}, E_{L}\right)$ определим ВЦФ:

$$
\begin{aligned}
& F(X)=\left\{F(x)=\left(F_{\downarrow} 1(x), F_{\downarrow} 2(x), F_{\downarrow} 3(x), F_{\downarrow} 4(x), F_{\downarrow} 5(x)\right), x \in X\right. \\
& F_{1}(x)=\sum_{e \in E_{x}} w(e) \rightarrow \min
\end{aligned}
$$

$\sum_{\text {где } е E_{x}} w(e)-$ общий вес покрытия $x$;

$$
F_{2}(x)=\min _{k=1, K} w\left(C_{k}\right) \rightarrow \max
$$

где $C_{k}$ - максимальна цепь, $k=1, K$, из покрытия $x \in\left\{C_{1}, C_{2}, \ldots, C_{k}, \ldots, C_{K}\right\}$, а $w\left(C_{k}\right)$ - ее длина (суммарный вес ребер цепи).

$$
F_{3}(x)=N(x) \rightarrow \min
$$

где $N(x)$ - число всех максимальных цепей в покрытии $x$;

$$
F_{4}(x)=i \rightarrow \min
$$

для всякой смешенной цепи $C^{i}$ из покрытия $x$.

$$
F_{5}(x)=\left|\rho_{x}(u, v)-\rho_{G_{L}}(u, v)\right| \rightarrow \min \equiv
$$


где для любых вершин $u, v \in G_{L}$ графа $\rho_{x}(u, v)$ - расстояние в покрытии $x$, a $\rho_{G_{L}}(u, v)$ - расстояние на графе $G_{L}$;

Все покрытия $\{x\}$ предфрактального графа $G_{L}$ образуют множество допустимых решений $X=X\left(G_{L}\right)=\{x\}$ векторно-целевой функции $(1)-(6)$.

В понятиях транспортных систем приведенные критерии векторноцелевой функции (1) имеют определенную содержательную интерпретацию.

Критерий (2) учитывает затраты пассажиров и администрации транспортной системы. При эксплуатации расходы должны быть минимальны.

Критерий (3) отражает нахождение маршрутов пассажирского транспорта с наибольшим количеством узлов на своем пути. Оптимальным для этого критерия является покрытие, содержащее максимальные цепи.

Чтобы доехать до нужного узла транспортной системы с наименьшим числом пересадок, необходимо уменьшить общее количество маршрутов в системе. На это направлен критерий (4).

Важными особенностями транспортной системы считаются локальность и дифференциация ее маршрутов. Внутрирегиональными (городскими, внутрирайонными) должны быть транспортные маршруты меньшей длины и меньшего веса, тем самым обеспечивая локальность. Таким образом, упрощается процесс администрирования транспортной системой на определенном уровне (района, города и т.д.). Межрегиональными являются маршруты более длинные и с большим весом. Под дифференциацией понимается разделение маршрутов по их функциям на межрегиональные и внутрирегиональные. При пересечении внутрирегиональности и межрегиональности может произойти нарушение дифференциации, т.е. ухудшение в функциональности маршрута. За недопущение таких ситуаций в работе транспортной системы в векторно- 
целевой функции (1) отвечает критерий (5). Смешанная цепь $C_{k}$ есть модель маршрута, сочетающая в себе обе функции - внутрирегиональную и межрегиональную. Так как ее старые ребра соединяют блоки и подграфзатравки предфрактального графа $G_{L}$, которые и соответствуют картам дорог районов, городов и т.д.

При эксплуатации транспортной системы часто требуется добраться до конечного пункта с наименьшим количеством остановок. Критерий (6) отражает эти требования к построению таких маршрутов.

Весам ребер предфрактального графа $G_{L}$, могут соответствовать определенные затраты и ограничения при движении транспорта по узлам транспортной системы.

\section{Заключение}

Формально численным решением индивидуально задачи является нахождение MA $X^{\uparrow}$ * $X$ из множества допустимых решений. В широком смысле под решением задачи понимается построения определенного алгоритма, гарантирующего нахождение МА для всякой индивидуальной задачи.

В последующих работах будут построены и обоснованы для решения рассматриваемой модельной задачи алгоритмы с оценками эффективности $[11,12]$.

\section{Литература}

1. Горев А. Э. Основы теории транспортных систем: учеб. пособие / А. Э. Гореев; СПбГАСУ. - СПб., 2010. - 214 с.

2. М. Гэри. Вычислительные машины и труднорешаемые задачи / Гэри М., Джонсон Д. - М.: Мир, 1982.

3. Павлов Д. А. Моделирование крупномасштабной транспортной сети предфрактальными графами / Павлов Д.А. // Политематический сетевой электронный научный журнал Кубанского государственного аграрного университета (Научный журнал КубГАУ) [Электронный ресурс]. - Краснодар: КубГАУ, 2017. №07(131). - Режим доступа:http://ej.kubagro.ru/2017/07/pdf/85.pdf.

4. Кочкаров А. М. Распознавание фрактальных графов. Алгоритмический подход / А.М. Кочкаров. - Нижний Архыз: Изд. центр «CYGNUS», 1998. - 170 с. 
5. Кроновер Р. М. Фракталы и хаос в динамических системах. Основы теории. Пер. с англ. - М.: Изд-во Постмаркет, 2000. - 352 с.

6. Кочкаров А. М. Структурная динамика и исследование структурновременных характеристик дискретных систем / А. М. Кочкаров, А. А. Кочкаров, С. П. Никищенко // Известия ТРТУ. Тематический выпуск "Перспективные системы и задачи управления". - Таганрог: ТРТУ, 2006. - № 3. - С. 235-238.

7. Лекции по теории графов / В.А. Емеличев, О.И. Мельников, В.И. Сарванов, Р.И. Тышкевич. - М. : Наука, 1990. - 383 с.

8. Перепелица В. А. Многокритериальные модели и методы для задач оптимизации на графах / В. А. Перепелица // LAP LAMBERT Academic Publication, 2013. - 333 c.

9. Кочкаров Р. А. Многовзвешенные предфрактальные графы с недетерминированными весами. Приложения в экономике, астрофизике и сетевых коммуникациях / Р. А. Кочкаров. - М.: ЛЕНАНД, 2017. - 432 с.

10. Цвиркун А.Д. Управление развитием крупномасштабных систем в новых условиях // Проблемы управления. - 2003. - №1 - С. 34-43

11. Павлов Д.А. Многокритериальная задача выделения маршрутов на предфрактальном графе / Д. А. Павлов, С. И. Салпагаров // Известия ТРГУ. Таганрог : ТРГУ, 2004. - №8 (43). - С. 303-304.

12. Барановская Т. П. Моделирование крупномасштабных транспортных сетей с применение методов многокритериальной оптимизации и учетом структурной динамики / Т. П. Барановская, Д. А. Павлов // Политематический сетевой электронный научный журнал Кубанского государственного аграрного университета (Научный журнал КубГАУ) [Электронный ресурс]. - Краснодар: КубГАУ, 2016. - №06(120). С. 1686 - 1705. - IDA [article ID]: 1201606111. - Режим доступа: http://ej.kubagro.ru/2016/06/pdf/111.pdf.

\section{References}

1. Gorev A. Je. Osnovy teorii transportnyh sistem: ucheb. posobie / A. Je. Goreev; SPbGASU. - SPb., 2010. - 214 s.

2. M. Gjeri. Vychislitel'nye mashiny i trudnoreshaemye zadachi / Gjeri M., Dzhonson D. - M.: Mir, 1982.

3. Pavlov D. A. Modelirovanie krupnomasshtabnoj transportnoj seti predfraktal'nymi grafami / Pavlov D.A. // Politematicheskij setevoj jelektronnyj nauchnyj zhurnal Kubanskogo gosudarstvennogo agrarnogo universiteta (Nauchnyj zhurnal KubGAU) [Jelektronnyj resurs]. - Krasnodar: KubGAU, 2017. - №07(131). - Rezhim dostupa:http://ej.kubagro.ru/2017/07/pdf/85.pdf.

4. Kochkarov A. M. Raspoznavanie fraktal'nyh grafov. Algoritmicheskij podhod / A.M. Kochkarov. - Nizhnij Arhyz: Izd. centr «CYGNUS», 1998. - 170 c.

5. Kronover R. M. Fraktaly i haos v dinamicheskih sistemah. Osnovy teorii. Per. s angl. - M.: Izd-vo Postmarket, 2000. - 352 s.

6. Kochkarov A. M. Strukturnaja dinamika i issledovanie strukturno-vremennyh harakteristik diskretnyh sistem / A. M. Kochkarov, A. A. Kochkarov, S. P. Nikishhenko // Izvestija TRTU. Tematicheskij vypusk "Perspektivnye sistemy i zadachi upravlenija". Taganrog: TRTU, 2006. - № 3. - S. 235-238.

7. Lekcii po teorii grafov / V.A. Emelichev, O.I. Mel'nikov, V.I. Sarvanov, R.I. Tyshkevich. - M. : Nauka, 1990. - 383 s.

8. Perepelica V. A. Mnogokriterial'nye modeli i metody dlja zadach optimizacii na grafah / V. A. Perepelica // LAP LAMBERT Academic Publication, 2013. - 333 s. 
9. Kochkarov R. A. Mnogovzveshennye predfraktal'nye grafy $s$ nedeterminirovannymi vesami. Prilozhenija $\mathrm{v}$ jekonomike, astrofizike i setevyh kommunikacijah / R. A. Kochkarov. - M.: LENAND, 2017. - $432 \mathrm{~s}$.

10. Cvirkun A.D. Upravlenie razvitiem krupnomasshtabnyh sistem $\mathrm{v}$ novyh uslovijah // Problemy upravlenija. - 2003. - №1. - S. 34-43

11. Pavlov D. A. Mnogokriterial'naja zadacha vydelenija marshrutov na predfraktal'nom grafe / D. A. Pavlov, S. I. Salpagarov // Izvestija TRGU. - Taganrog : TRGU, 2004. - №8 (43). - S. 303-304.

12. Baranovskaja T. P. Modelirovanie krupnomasshtabnyh transportnyh setej s primenenie metodov mnogokriterial'noj optimizacii i uchetom strukturnoj dinamiki / T. P. Baranovskaja, D. A. Pavlov // Politematicheskij setevoj jelektronnyj nauchnyj zhurnal Kubanskogo gosudarstvennogo agrarnogo universiteta (Nauchnyj zhurnal KubGAU) [Jelektronnyj resurs]. - Krasnodar: KubGAU, 2016. - №06(120). S. 1686 - 1705. - IDA [article ID]: 1201606111. - Rezhim dostupa: http://ej.kubagro.ru/2016/06/pdf/111.pdf. 